BUHEP-96-42

September 1996

\title{
Critical properties and monopoles in $U(1)$ lattice gauge theory
}

\author{
Werner Kerler ${ }^{a}$, Claudio Rebbi ${ }^{b}$, Andreas Weber ${ }^{a}$ \\ ${ }^{a}$ Fachbereich Physik, Universität Marburg, D-35032 Marburg, Germany \\ ${ }^{b}$ Department of Physics, Boston University, Boston, MA 02215, USA
}

\begin{abstract}
We present a detailed study of the properties of the phase transition in the four-dimensional compact $\mathrm{U}(1)$ lattice gauge theory supplemented by a monopole term, for values of the monopole coupling $\lambda$ such that the transition is of second order. By a finite size analysis we show that at $\lambda=0.9$ the critical exponent is already characteristic of a second-order transition. Moreover, we find that this exponent is definitely different from the one of the Gaussian case. We further observe that the monopole density becomes approximately constant in the second-order region. Finally we reveal the unexpected phenomenon that the phase transition persists up to very large values of $\lambda$, where the transition moves to (large) negative $\beta$.
\end{abstract}




\section{Introduction}

The continuum limit can be recovered from the lattice regularization of a quantum field theory by approaching a critical point with suitable scaling properties. While the existence of such a critical point can be established through perturbative considerations in the case of asymptotically free theories, very little is known about critical points that can give origin to continuum limits in the non-perturbative domain. The $U(1)$ lattice gauge theory, although characterized by the most elementary continuous gauge symmetry, has a rather complex phase structure, induced by the presence of monopole excitations. While QED, which shares the same gauge group, appears very successfully embedded in the $S U(2) \times U(1)$ theory of electroweak interactions, it is still quite interesting to study the properties of the pure $U(1)$ lattice gauge theory in view of what one can learn from the existence of a non-trivial critical point and its implications for possible continuum limits of lattice gauge theories in the non-perturbative domain.

We have devoted several investigations to the study of the properties of the $U(1)$ lattice gauge theory, taking special advantage of a technique we introduced to bridge the valley between energy gaps [1], 2], and of a new order parameter based on the topological properties of monopole networks [1, 3]. Our investigations have been based on the study of the model with the Wilson action supplemented by an additional monopole term [4]

$$
S=\beta \sum_{\mu>\nu, x}\left(1-\cos \Theta_{\mu \nu, x}\right)+\lambda \sum_{\rho, x}\left|M_{\rho, x}\right|
$$

where $M_{\rho, x}=\epsilon_{\rho \sigma \mu \nu}\left(\bar{\Theta}_{\mu \nu, x+\sigma}-\bar{\Theta}_{\mu \nu, x}\right) / 4 \pi$ and the physical flux $\bar{\Theta}_{\mu \nu, x} \in[-\pi, \pi)$ is related to the plaquette angle $\Theta_{\mu \nu, x} \in(-4 \pi, 4 \pi)$ by $\Theta_{\mu \nu, x}=\bar{\Theta}_{\mu \nu, x}+2 \pi n_{\mu \nu, x}$ [5].

It is well known that this system undergoes a phase transition at a coupling $\beta_{\mathrm{cr}}(\lambda)$ separating a disordered phase, characterized by a condensate of monopole excitations, for $\beta<\beta_{\mathrm{cr}}$, from an ordered, Coulomb-like phase for $\beta>\beta_{\mathrm{cr}}$. Our own 
studies of the energy distribution have shown that as $\lambda$ increases the phase transition changes nature: a gap in the energy distribution indicates that the transition is of the first order for $\lambda=0$ and for moderately small values of $\lambda$, whereas the disappearance of the gap for larger values of $\lambda$ indicates that the transition becomes there of the second order. In this paper we wish to contribute further to the knowledge of the $U(1)$ lattice gauge theory by exploring in detail the region of larger $\lambda$ couplings, where the phase transition appears to be continuous. An important aim of our investigation is to verify by a finite size analysis that the transition is indeed of second order and to determine deviations of the critical exponent from the Gaussian case. A further goal is to clarify the behavior of the transition when $\lambda$ becomes very large. Because the phase transition is so closely related to the appearance of a monopole condensate, exploring in detail the effects of the monopole term in the action appears to be of particular interest.

In this study we use periodic boundary conditions, guaranteeing translational invariance and homogeneity.

\section{The line of phase transitions}

In the paper where they introduced the action (1.1) Barber and Shrock already made the observation that there is a shift of the transition point if $\lambda$ is varied [4]. It has also been reported in the literature [6, 7] that a complete suppression of monopoles, corresponding to $\lambda=\infty$, produces the disappearance of the confining phase. In our previous investigations we determined the location of the phase transition $\beta_{\mathrm{cr}}(\lambda)$ for a wide range of values of $\lambda[1,2]$. Now we wish to clarify what happens at very large $\lambda$. For this purpose we determine the critical points at values of $\lambda$ substantially larger than previously considered and if necessary, allow $\beta$ to become negative.

In order to keep the computational cost for this study within bearable limits we have used our topological characterization of the phases [1], 3] to locate the critical 
points. Our classification is based on the fact that there is an infinite network of monopole current lines in the confining phase and no such network in the Coulomb phase. On finite lattices "infinite" is to be defined in accordance with the boundary conditions [ 8 ]. For the periodic boundary conditions we are considering here, "infinite" is equivalent to "topologically nontrivial in all directions". While for loops the topological characterization is straightforward, to determine the existence of an infinite network it is necessary to perform a more elaborate analysis based on homotopy preserving mappings [1, 3].

Because the probability $P_{\text {net }}$ to find a network which is nontrivial in all directions takes values exactly 1 and 0 in the confining phase and in the Coulomb phase, respectively, the frequency of the occurrence of such a network is a very advantageous order parameter. Indeed, with an infinite system a single configuration would be sufficient to identify the phase. On a finite lattice, because of finite size effects, the distinction is not as sharp, but still few configurations are sufficient to discriminate bewteen the phases. Examples are given in Figure 1.

It should be remembered that on finite lattices different order parameters lead to slightly different critical $\beta$. On an $8^{4}$ lattice the maximum of the specific heat and our topological order parameter give values 1.0075(1) and 1.0074(2) for $\lambda=0$, and $0.3870(5)$ and $0.372(3)$ for $\lambda=0.9$, respectively. To determine the location of the maximum of the specific heat for larger $\lambda$ in an efficient way we first determine the critical $\beta$ from the topological order parameter and then find the maximum of the specific heat in an easy second step.

In Figure 2 we show our results for the location of the phase transition $\beta_{\mathrm{cr}}$, defined by the maximum of the specific heat, for values of $\lambda$ ranging up to 1.3 . It can be seen that the phase transition line continues to negative $\beta$.

Using the topological order parameter we could follow the line of phase transitions up to still much larger $\lambda$ : from $\lambda=1.4$ where $\beta_{\text {cr }}=-0.52(2)$ to $\lambda=10$ where $\beta_{\text {cr }}$ is approximately -1000 . It should be emphasized that the characteristic topological signatures for the two phases are apparent throughout entire range of $\lambda$. This shows 
that, quite remarkably, both phases persist all the way up to very large $\lambda$.

We find that finite size effects increase with $\lambda$. This is indicated by the fact that the transition region becomes broader. From Figure 3 it can be seen that the width of the peak of the specific heat increases and that its height decreases with increasing $\lambda$. Obviously for very large $\lambda$ a precise determination of the location of the maximum of the specific heat becomes quite difficult. The increase of the width of the transition region with $\lambda$ is also apparent from the data on the topological order parameter $P_{\text {net }}$ in Figure 1 (notice the different scales for $\beta$ in the upper and lower parts of the figure). Still, as we already remarked above, the fact that the topological order parameter takes values 0 and 1 in the two phases makes it easy to discriminate between the two phases even at very large $\lambda$.

\section{Critical behavior}

In order corroborate our observation that for large $\lambda$ the phase transition becomes of second order we have investigated the finite-size scaling behavior of the maximum of the specific heat $C_{\max }$. It is expected to be

$$
C_{\max } \sim L^{d}
$$

if the phase transition is of first order and

$$
C_{\max } \sim L^{\frac{\alpha}{\nu}}
$$

if it is of second order, where $\alpha$ is the critical exponent of the specific heat and $\nu$ the critical exponent of the correlation length.

In Figure 4 we present results of our simulations for $C_{\max }$, on lattices with $L=6$, 8, 10, 12 for $\lambda=0.9$ at the corresponding values of $\beta_{\mathrm{cr}}$. The fit to these data gives

$$
\frac{\alpha}{\nu}=0.485(35)
$$

Clearly this is quite far from 4 and thus the transition not of first order. 
From the hyperscaling relation $\alpha=2-d \nu$ we find

$$
\nu=0.446(5)
$$

It is interesting to note that (3.5) is clearly different from the value $\frac{1}{2}$ of the Gaussian case.

The critical $\beta$ is expected to behave as

$$
\beta_{\mathrm{cr}}(L)=\beta_{\mathrm{cr}}(\infty)+a L^{-\frac{1}{\nu}}
$$

From this relation, using the value in Eq. (3.5) and our data for $\beta_{\text {cr }}(L)$ at $\lambda=0.9$, we get $\beta_{\mathrm{cr}}(\infty)=0.4059(5)$ and $a=-1.99(6)$.

We have also performed simulations for $\lambda=0.8$ on lattices with $L=6,8$, 10. It turns out that $C_{\max }$ does not yet scale in this case. This might be related to the particular sensitivity close to a tricritical point.

In Figure 5 we present the monopole density $\rho=\left(1 / 4 L^{4}\right) \sum_{\rho, x}\left|M_{\rho, x}\right|$ along the transition line. It is interesting to observe that starting at $\lambda=0.9$ the density becomes constant within the errors of the simulation.

\section{Discussion}

In the past values between 0.33 and 0.50 have been found for the critical exponent $\nu$, in different contexts [9, [10]. This wide range of values has prevented firm conclusions. More precise results, based on a finite size analysis and higher statistics, have been recently reported [11] for the Wilson action extended by a double charge term with coupling $\gamma$.

The well known features of this action are that the first order transition, which occurs at $\gamma=0$, weakens with increasing $\gamma$ until it becomes of second order at a tricritical point. While for the usual periodic boundary conditions the second order region starts at negative $\gamma$, the authors of Ref. [11], using a spherelike lattice, find evidence for a second order transition already at $\gamma=0$. Of course, this could also be 
due to the fact that inhomogeneities weaken the transition [8] and that the mapping from the surface of a hypercube to the 4-sphere can provide inhomogeneities.

The result $\nu=0.446(5)$ presented here and the result $\nu=0.365(8)$ given in Ref. [11] are both definitely different from the value $\frac{1}{2}$ of the Gaussian case. Thus a continuum limit of four-dimensional pure $\mathrm{U}(1)$ gauge theory, nontrivial and not asymptotically free, appears possible.

The difference between the two results, though not dramatic, is too large to be accounted for by systematic errors. Further studies appear necessary. In particular, simulations at some value of $\lambda$ larger than 0.9 will be important for seeing whether the value of Eq. (3.5) is universal. Some of the results obtained in Ref. 111 for three different values of $\gamma$ and using different techniques, though close to each other, also differ beyond the statistical errors. Results for yet more negative $\gamma$ and also for periodic boundary conditions would desirable.

The increase of the finite-size effects with lambda mentioned in Sect. 2 could hint at an increase of $\nu$, related to a larger correlation length $\eta \sim\left|\beta-\beta_{\mathrm{cr}}\right|^{-\nu}$. The corresponding decrease of $\alpha$ from $0.216(16)$ at $\lambda=0.9$ to 0 , in view of the relation $C \sim\left|\beta-\beta_{\mathrm{cr}}\right|^{-\alpha}$, also appears consistent with the behavior of the specific heat seen in Figure 3. The behavior of $\alpha$, in particular, lends support to the fact that the increase of finite-size effects is due to the exponents and not to the constants in front of the scaling expressions. Possibly $\nu$ could reach in this way the Gaussian value.

In regard to our result that both phases are still present for very large $\lambda$, where $\beta_{\mathrm{cr}}$ becomes negative, it is to be noted that quite some time ago a similar phenomenon has been observed for vortex strings in the 3D $\mathrm{O}(2)$ spin model [12]. It should be further mentioned that negative $\beta$ have also been studied in the compact $\mathrm{U}(1)$ lattice gauge with complete suppression of monopoles and inclusion of matter fields, with the observation of a first order transition at $\beta=-0.7$ 13, 14. Clearly this is far away from our phase transition line which for very large $\lambda$ runs at extremely negative $\beta$.

We have already shown in Figure 7 of Ref. [1] that along the phase transition 
line the average plaquette energy $E=\left(1 / 6 L^{4}\right) \sum_{\mu>\nu, x}\left(1-\cos \Theta_{\mu \nu, x}\right)$ increases with $\lambda$. This increase continues for still larger $\lambda$ so that ultimately one comes close to the upper limit of the range $0 \leq E \leq 2$. Thus negative plaquettes contribute significantly. In this context it should be noted, however, that, even for $\lambda=\infty$, for sufficiently negative $\beta$ the complete suppression of negative plaquettes only reduces $E$ by roughly a factor 2 [13].

The non-Gaussian exponent we found implies that the continuum limit of our model is different from the usual expectations for QED and would not be seen in perturbative approaches. Possibly monopoles survive in the limit. One should also be aware of the possibility of obtaining different limits when approaching from the strong or the weak coupling regions 15 .

As a matter of fact, we most likely have a line of critical points in $(\beta, \lambda)$ space at which different continuum limits may arise if $\nu$ is not universal. This line is to be distinguished from the line of critical points for $\beta>\beta_{\text {cr }}$ in case of the pure Wilson action, whose existence has been conjectured a long time ago. There the scale invariance of Creutz ratios [16] hints at a diverging correlation length and thus at a line of critical points. Similar indications come from calculations of the spectrum [17].

Further work appears necessary to fully explore the critical properties of the model and, in particular, to determine whether its critical points can give origin to a viable theory. A related question is which form of the action is the most profitable to use. The addition of a monopole term is attractive because of the close relation of monopoles to the phase structure. We wish to note, however, that instead of $\lambda \sum_{\rho, x}\left|M_{\rho, x}\right|, \mu \sum_{\rho, x}\left(M_{\rho, x}\right)^{2}$ or some other form might turn out to give better results.

\section{Acknowledgments}

This research was supported in part under DFG grants Ke 250/7-2 and 250/12-1 and under DOE grant DE-FG02-91ER40676. 


\section{References}

[1] W. Kerler, C. Rebbi and A. Weber, Phys. Rev. D 50 (1994) 6984.

[2] W. Kerler, C. Rebbi and A. Weber, Nucl. Phys. B 450 (1995) 452.

[3] W. Kerler, C. Rebbi and A. Weber, Phys. Lett. B 348 (1995) 565.

[4] J.S. Barber and R.E. Shrock, Nucl. Phys. B 257 (1985) 515.

[5] T. DeGrand and D. Toussaint, Phys. Rev. D 22 (1980) 2478.

[6] J.S. Barber, R.E. Shrock and R. Schrader, Phys. Lett. B 152 (1985) 221.

[7] V.G. Bornyakov, V.K. Mitrjushkin and M. Müller-Preussker, Nucl. Phys. B (Proc. Suppl.) 30 (1993) 587.

[8] W. Kerler, C. Rebbi and A. Weber, Phys. Lett. B 380 (1996) 346.

[9] R. Gupta, M.A. Novotny and R. Cordery, Phys. Lett. B 172 (1986) 86, and references therein.

[10] C.B. Lang, Nucl. Phys. B 280 (1987) 255, and references therein.

[11] J. Jersák, C.B. Lang and T. Neuhaus, Phys. Rev. Lett. 77 (1996) 1933; Phys. Rev. D, to be published.

[12] G. Kohring and R.E. Shrock, Nucl. Phys. B 288 (1987) 397.

[13] A. Hoferichter, V.K. Mitrjushkin and M. Müller-Preussker, Phys. Lett. B 338 (1994) 325.

[14] Balasubramanian Krishnan, U.M. Heller, V.K. Mitrjushkin and M. MüllerPreussker, preprint HU Berlin-IEP-96/16.

[15] M. Creutz, Phys. Rev. D 23 (1981) 1815.

[16] G. Bhanot, Phys. Rev. D 24 (1981) 461.

[17] B. Berg and C. Panagiotakopoulos, Phys. Rev. Lett. 52 (1984) 94. 


\section{Figure captions}

Fig. 1. Order parameter $P_{\text {net }}$ as function of $\beta$ for $\lambda=0$ and $\lambda=0.9$ on an $8^{4}$ lattice.

Fig. 2. Location of the phase transition point $\beta_{\mathrm{cr}}$ as function of $\lambda$ for $8^{4}$ (squares) and $16^{4}$ (crosses) lattices.

Fig. 3. Specific heat $C$ as function of $\beta$ for $\lambda=0,0.9,1.3$ on an $8^{4}$ lattice.

Fig. 4. $C_{\max }$ versus $L$ for $\lambda=0.9$ at $\beta_{\text {cr }}$.

Fig. 5. Monopole density as function of $\lambda$. 

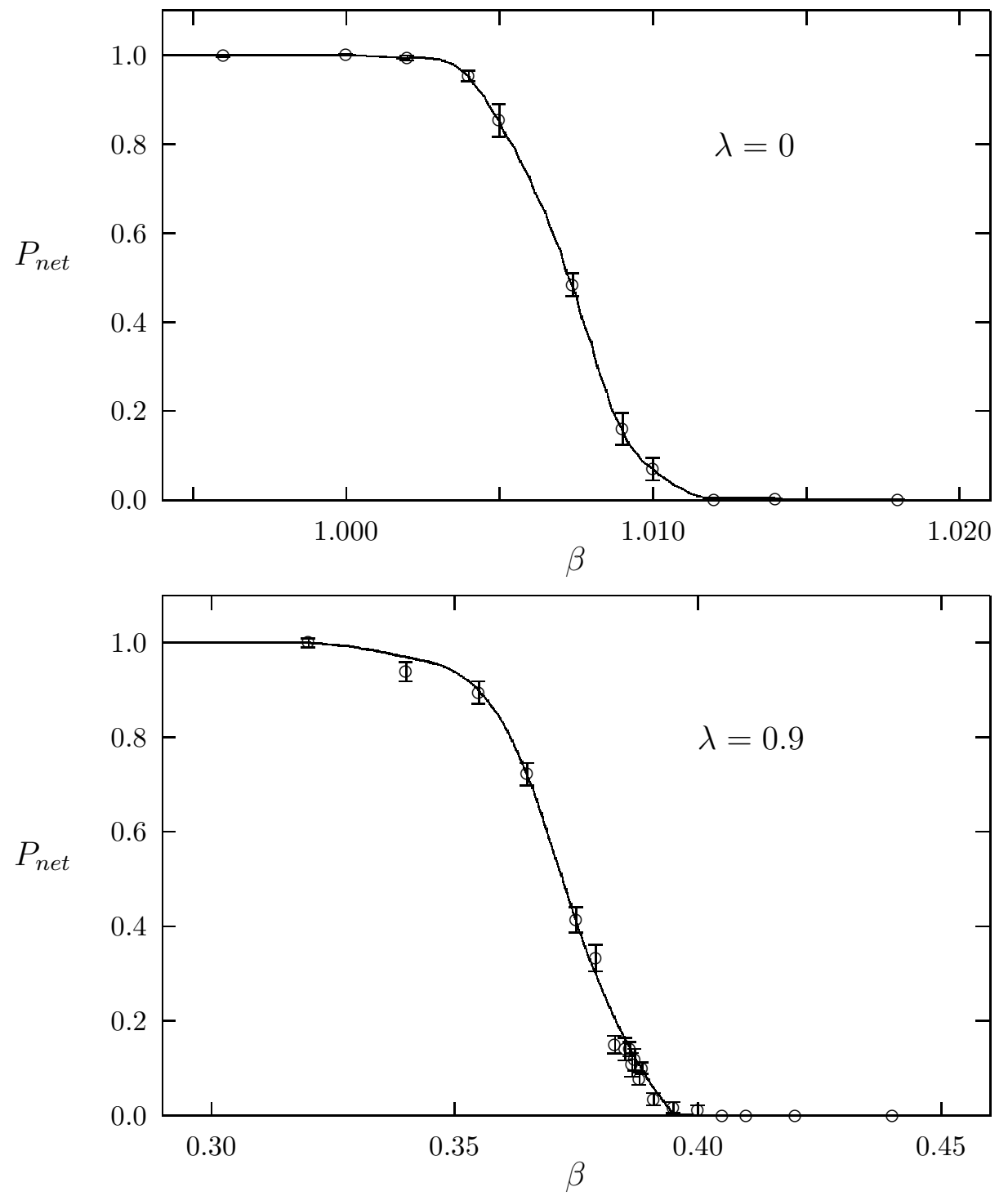

Figure 1: 


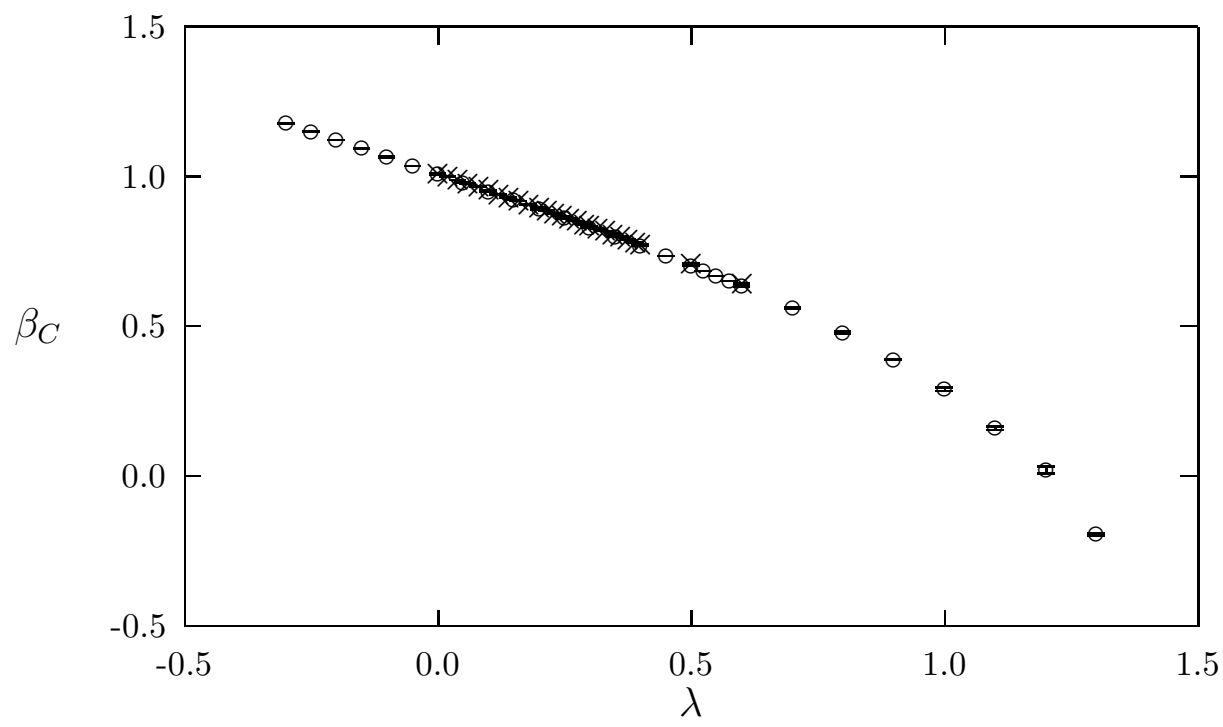

Figure 2: 

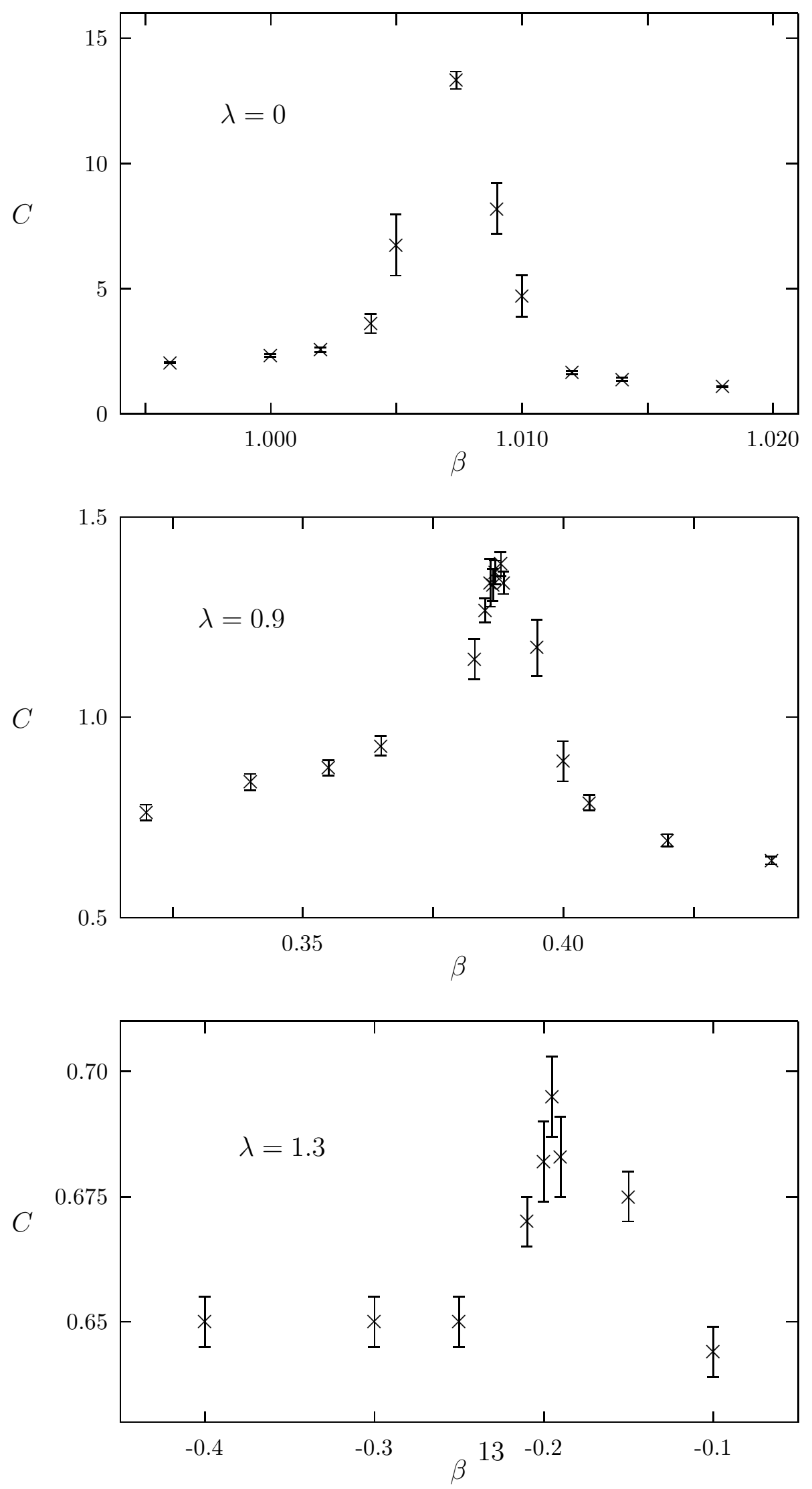

Fionure 3. 


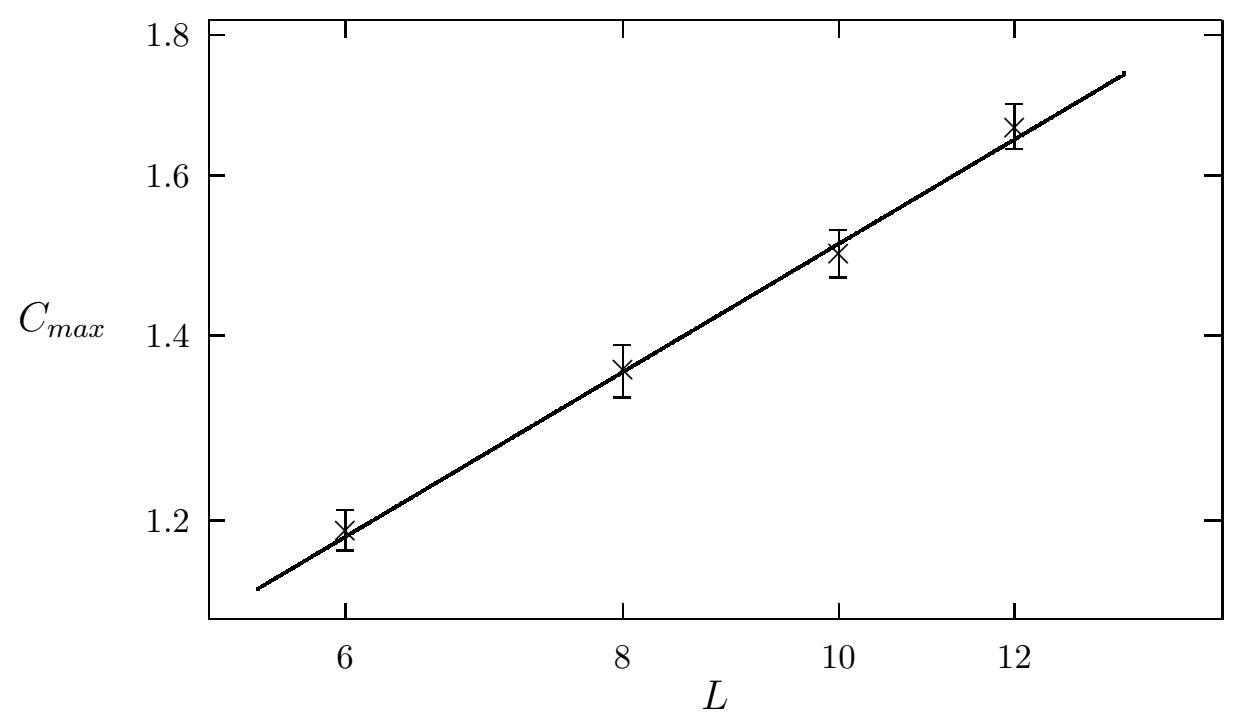

Figure 4:

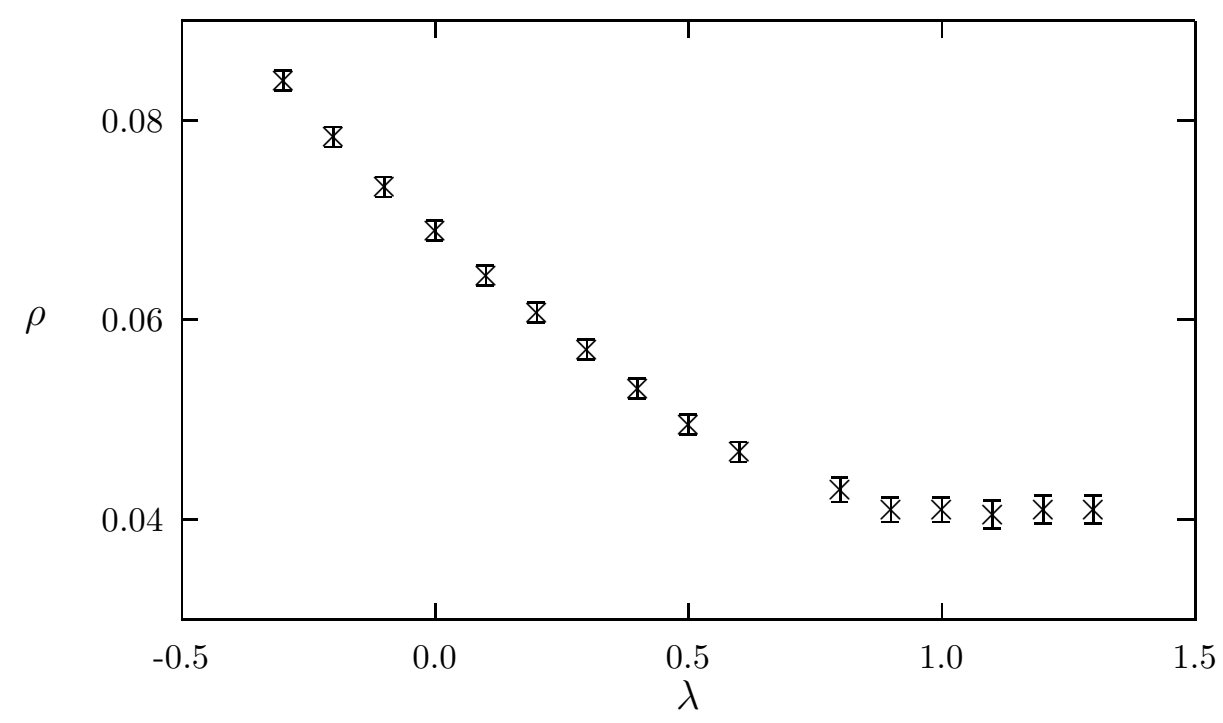

Figure 5: 